\title{
LA MUJER Y LA TRADUCCIÓN EN LA REPÚBLICA FEDERAL ALEMANA ¿UNA VOCACIÓN NATURAL O FORZADA?
}

\author{
Eva Parra Membrives
}

\begin{abstract}
An enquiry performed in the year 1985 in the Federal Republic of Germany by Silbermann and Hänseroth revealed that a surprisingly high percentage of the German population thought that traduction was an activity of lower social importance, which should be mainly developed by women. As this seems to implicate that German women had to reduce themselves to activities of subordinate social interest, this essay tries to discover the possible historic origin of this belief and investigates if, in ages as distant as the Middle Ages, women and traduction were in fact intimately related because of their social triviality, and if there is to find a connection even today between social insignificance and female traduction.
\end{abstract}

En el año 1985 lós investigadores alemanes Alphons Silbermann y Albin Hänseroth realizan una encuesta por las calles de Colonia, República Federal de Alemania, que tenía por objeto descubrir de qué imagen disfrutaba el traductor como profesional en la opinión pública alemana. Se trataba de comprobar una idea latente en ambos quie les sugería que

"[...] Ohne die Tätigkeit des Übersetzens, jede zeitgenössische Gesellschaft funktionsunfähig würde, eine Feststellung, die die Vermutung impliziert - und deshalb wird sie an dieser Stelle geäußert -, daß die Tätigkeit des Übersetzers eine vergleichsweise mit hohem Berufs- und Sozialprestige ausgestattete sein mu $\beta^{\text {“I }}$

Sorprendidos, han de constatar sin embargo que precisamente tiene lugar aquí el fenómeno contrario. En el sondeo por ellos efectuado había de diferenciarse de entre una relación de 50 profesiones o actividades diferentes cuáles eran tenidas, con vistas al futuro, por profesiones o actividades muy importantes, cuáles de ellas se conceptuaban de menor importancia, y cuáles podían considerarse según este parámetro sin importancia en absoluto. Una vez analizados los resultados, Silbermann y Hänseroth comprueban que nada menos que un $80 \%$ de las respuestas sitúa a la traducción entre las profesiones de menor importancia. Un 7\% adicional, incluso, entre las actividades totalmente carentes de ella. Para justificar sus opiniones, los encuestados citaban como razón principal que en la

\footnotetext{
${ }^{1}$ A. Silbermann; A. Hänseroth, Der Übersetzer. Eine berufs-und literatursoziologische Untersuchung, (Wiesbaden 1985) 2
} 
actualidad la población en general domina cada vez más lenguas extranjeras, por lo cual podrá prescindirse en el futuro de traducciones y traductores.

El resultado arrojado por esta primera cuestión llevó a los autores de la investigación a formular una segunda pregunta. Se trataba ahora de averiguar si este mismo público no directamente relacionado con la traducción contemplaba a ésta como una profesión o actividad preferentemente masculina, femenina o de carácter indiferente. De nuevo, el acuerdo popular produce una coincidencia en las respuestas que provoca que se alcancen cifras bastante uniformes en un porcentaje muy similar al anterior, ya que las contestaciones arrojan como resultado que nada menos que a un $75 \%$ les parecen mucho más adecuadas las traductoras de sexo femenino. Para justificar este pensamiento, los encuestados no pueden ofrecer ningún dato tangible, pues ninguno de ellos logró recordar o citar algún caso concreto por él conocido, en persona o por referencias, de una mujer que se dedicase profesionalmente a la traducción, y que por lo tanto le pudiese parecer en especial apta para ello. Los motivos que les habían llevado a considerar la traducción como una actividad preferentemente de mujeres habían sido en realidad características que consideraban propias e inmanentes a la actividad traductora: definían la traducción como una labor que exigía una alta capacidad de intuición, mucha paciencia, y permitía poca fantasía creativa. Al parecer, para los encuestados, exigencias a las que una mujer se hallaba en mejor disposición de adaptarse.

Sin embargo, a partir de esta encuesta, sus autores creyeron haber descubierto una explicación bien distinta para la adscripción a un determinado sexo de la traducción, una justificación íntimamente relacionada con las perspectivas de futuro de la traducción como profesión, y que podría considerarse consecuencia lógica de éstas, tal como queda definida en las palabras siguientes:

"Die Unsichtbarkeit des Berufes in der Öffentlichkeit und die geringe Bedeutungszuweisung berücksichtigend, ist es nicht sonderlich überraschend, daß nahezu 75\% der Befragten Übersetzen als typische Frauentätigkeit ansahen"2

Obsérvese que esto ha de significar que, según estos investigadores, el acceso preferente de la mujer a la traducción en la sociedad alemana actual vendría dado por ser considerada esta actividad en particular una ocupación marginal y menor, hecho, que llevaría además implícita la suposición de que la mujer tradicionalmente se ha de ocupar de actividades sociales menos importantes. Lo peligroso de esta afirmación es evidente, pero, a la vez, pareció aquí de sumo interés comprobar lo fundamentado de este pensamiento.

La primera parte de la aseveración de estos investigadores, que afecta a la traducción, no parece demasiado aventurada, ya que su escaso prestigio social había ya quedado bien patente a partir de las perspectivas de futuro que la población le había asignado en la encuesta de Silbermann y Hänseroth, así como en el justificar los encuestados sus respuestas con que, con el tiempo, al generalizarse el dominio de varios idiomas, el

\footnotetext{
${ }^{2}$ A. Silbermann, op. cit., 4
} 
traductor se convertiría en superfluo. Además, otras cuestiones que interesaron posteriormente a Silbermann y Hänseroth, como, por ejemplo, qué grado de preparación exigiría la traducción como actividad, lograron confirmar que a la traducción se le atribuía popularmente, por lo general, una importancia más bien escasa. Incluso de la invisibilidad del traductor es posible apercibirse al observar cómo, a pesar de que los encuestados estiman que la traducción es una profesión femenina, son incapaces de recordar un solo nombre en concreto. Para comprobar, no obstante, el alcance que posee exactamente ese anonimato del traductor, es la estadística quien de verdad aporta datos concluyentes.

Entre los años 1966 y 1981, las traducciones al alemán han supuesto en el mercado editorial de la República Federal de Alemania un 10,9\% de las publicaciones totales, lo cual supone aproximadamente unos 5000 libros por año ${ }^{3}$. Esta cifra se mantiene más o menos estable desde el año 1975, con una ligera tendencia a incrementarse. Lamentablemente, no se disponen aquí de datos estadísticos más recientes, pero es de suponer que, en cuanto al número, las obras actualmente traducidas en la República Federal de Alemania habrán de ser similares o incluso superiores, si se tiene en cuenta la absorción de todas aquellas publicaciones surgidas en lo que antes era la República Democrática Alemana. Sin embargo, el catálogo informatizado de la 'Deutsche National Bibliothek', la Biblioteca Nacional Alemana, que contiene la relación de todas aquellas obras editadas en la República Federal de Alemania, menciona para el período comprendido entre los años 1986 y 1991 un total de 472 títulos publicados que incluyen en su referencia bibliográfica el nombre del traductor o traductora, o que simplemente advierten que se trata de una traducción. Un número bastante pobre, ya que, si se atiende a la estadística, deberían ser como mínimo alrededor de 30000, 5000 por cada año desde 1986 a 1991, las obras traducidas. La 'invisibilidad' de los 29528 traductores que no aparecen mencionados es patente, y las cifras parece que no necesitan de más comentarios.

Que estén probados el escaso prestigio social de la traducción y su anonimato no presupone, sin embargo, necesariamente, que se la deba considerar una profesión o actividad femenina por parte de la población, ya que no hay nada intrínseco a lo femenino que convierta sus actividades en más secretas por naturaleza. Si esto es efectivamente así, y hoy en día, cuando la igualdad teórica de la mujer es comúnmente aceptada y ésta se siente del todo emancipada, existe para la opinión pública una adecuación subconsciente de la mujer a la traducción a causa del escaso prestigio y la llamada invisibilidad de la segunda, debe poder hallarse algún motivo para ello, que probablemente encuentre su explicación en la historia de la mujer y, también quizá, en la de la traducción alemana.

Aquí, en aparente contradicción con la encuesta de Silbermann y Hänseroth, Friedmar Apel indica que

"Der oft zitierte Gemeinplatz, Deutschland sei das Land der Übersetzer, läßt sich an der Problemgeschichte sowohl qualitativ wie quantitativ erhärten"

\footnotetext{
${ }^{3}$ A. Silbermann, op. cit., $71 \mathrm{~s}$

${ }^{4}$ F. Friedmar: Literarische Übersetzung (Stuttgart: 1983) 38
} 
con lo que, en un principio, y si en Alemania se han producido no sólo estudios de calidad, sino además multitud de ellos, no debe parecer de tan escasa trascendencia la traducción en este país. Críticos como Günther Bärnthaler confirman además esta idea al asegurar que para el desarrollo de la literatura en Alemania a la traducción le corresponde un papel particularmente importante, pues

"Dem Übersetzen kommt für die Entwicklung der deutschsprachigen Literatur eine zentrale Funktion zu. Erst aus dem Übersetzen entstehen die althochdeutsche Literatur und in der Folge die volkssprachigen Literaturen überhaupt"

Esta función central que la traducción ha tenido, según Bärnthaler, para el desarrollo de la literatura alemana desde la Edad Media no implica sin embargo por necesidad que fuese históricamente, y para el hombre medieval que menciona el autor, una actividad socialmente prestigiosa, puesto que, como ya se ha visto antes, aunque hoy en día para el adecuado funcionamiento del mundo civilizado que se ha construido, la traducción resulta indispensable, la población en general no lo conceptúa de ese modo. Además, tendría que verse cómo valoraba el hombre medieval la literatura, y descubrir si ésta era socialmente prestigiosa en suficiencia como para que no sólo al autor, sino también al traductor literario le fuese reconocida una aportación social significativa.

En este sentido, de lo que, desde luego, no puede calificarse la labor de traducción durante la Edad Media es de invisible. No resulta nada difícil rastrear nombres de traductores en activo durante la Edad Media alemana, algunos de ellos incluso muy populares en su época, como Heinrich von Langenstein, Notker der Deutsche, Nikolaus von Dinkelsbühl, Leopold Stainreuther o Ulrich von Pottenstein. Y esta ausencia de secretismo puede aplicarse también a algunas traductoras de sexo femenino, como Hiltgart von Hürnheim, Ursula Satzenhofer, Elisabeth Kempf, Justina Blarerin o Anna Ebin' ${ }^{6}$, entre otras.

Desde el inicio de la historia de ambas, entonces, sí que es cierto que las mujeres y la traducción se han sentido unidas y han entrado en contacto, pero no en función de la invisibilidad de la traducción, que no era tal. De Elisabeth Kempf, una de las traductoras citadas hace un momento, dirán sus contemporáneos que

"Notabiliter erudita erat in scripturis, uti apparet in quam plurimis libris, quos de latino transtulit in vulgare...in quibus non pauca sororum provincie Theutonie monasteria consolantur..."7

Es evidente que si esta mujer se hubiese dedicado a traducir de forma oculta, sin mencionar a nadie el trabajo por ella realizado y su actividad fuese públicamente desconocida, su nombre no hubiera llegado hasta hoy. Además, en las palabras citadas hace unos instantes, puede apercibirse cierto grado de admiración y aprecio por la habilidad y la

\footnotetext{
${ }^{5}$ G. Bärnthaler, Übersetzen im deutschen Spätmittelalter. Der Mönch von Salzburg, Heinrich Laufenberg und Oswald von Wolkenstein als Übersetzer lateinischer Hymnen und Sequenzen (Göppingen 1983) 7

${ }^{6}$ Citados todos en: K. Ruh (ed.), Die deutsche Literatur des Mittelalters. Verfasserlexikon ( Berlin: 1978-)

${ }^{7}$ K.E. Geith, "Elisabeth Kempf”, en : K. Ruh (ed.), op. cit., T.4, 1115
} 
cultura de Elisabeth Kempf, destreza que se manifiesta únicamente en sus traducciones, pues, que se sepa, Elisabeth Kempf no ha compuesto ninguna obra literaria propia. Sin embargo, su actividad no sólo le ha permitido seguir siendo conocida siglos después de su muerte, sino que a ello, y sólo a ello, le debe su inclusión en el actualmente más importante catálogo de autores medievales alemanes, el Verfasserlexikon, diccionario de autores, iniciado por Wolfgang Stammler".

Es decir, que no sólo ha desaparecido el anonimato del traductor, sino que se le asimila al creador de un texto. Esto, que hoy en día puede resultar quizá desproporcionado y fuera de lugar, no lo es del todo, si se atiende a criterios medievales. El mundo medieval es final, no causal, y por lo tanto será necesario descubrir cuál es la función del autor de un texto, y cuál la del traductor de éste para ver si su equiparación resulta tan incongruente.

Como dijera Dirk Glogau utilizando una metáfora muy ilustrativa, el hombre medieval concibe el mundo que le rodea como si fuera un libro escrito por Dios ${ }^{9}$. Todo lo que en la realidad se encuentra es producto divino, y la función del arte será reproducir, en la medida de lo posible, la obra de Dios. Cualquier aportación personal del poeta será, en función a esto, irrelevante y, desde luego, no intencionadamente pretendida. Hennig Brinkmann asegura que:

"Und das darf jetzt schon gesagt werden, daß dem Mittelalter unmittelbar persönliche Aussprache nicht letzter Wert war" 10 .

El arte, y, como parte de él, la literatura, ya desde antes de que surgiese en mente de su autor ha sido, por la naturaleza de su origen, despojado de toda originalidad. Cualquier obra que el autor medieval decida escribir jamás habrá sido del todo construida por él, pues necesariamente habrá sido compuesta antes por Dios. En ese sentido, el autor literario no es creativo, sino que actúa como traductor, ya que ha trasladado un mensaje en concreto ya existente y elaborado previamente por Dios en una lengua muy particular a un sistema de signos humano del que pueden ser partícipes un mayor número de personas. El prestigio del autor viene dado por su capacidad de asimilar el idioma divino, por su competencia al dominar varios sistemas de comunicación de carácter tan dispar, el divino y el humano, y por su talento, si llega a ser capaz de utilizar sus habilidades tan adecuadamente como para obtener como resultado una obra artística o literaria. Pero nunca podrá pretender ser el autor material de lo que escriba.

De modo que, según la concepción medieval del mundo, pueden diferenciarse dos tipos distintos de traductores, pero no existe ningún autor al margen de Dios. De entre los traductores, habría que distinguir entre aquél, que traslada el mensaje divino a una lengua humana, conocido hoy en día como autor; y ese otro que lleva a cabo un paso más, el que

\footnotetext{
${ }^{8}$ Se trata de la ya citada obra de Kurt Ruh

9 "Die Welt als Buch Gottes" titula uno de los capítulos de su tesis D. Glogau, Untersuchungen zu einer konstruktivistischen Mediävistik: Tiere und Pflanzen im 'Tristan' Gottfrieds von Stra8burg und im Nibelungenlied (Essen: 1993)

${ }^{10}$ H. Brinkmann, Zu Wesen und Form mittelalterlicher Dichtung, (Tübingen: 1979) 10
} 
transporta el mismo mensaje divino ya traducido a una lengua humana previamente determinada a otra de similares características, es decir, el que traduce a partir de una traducción, pero moviéndose siempre dentro de sistemas de signos humanos. Ambos cumplen con una misma función: facilitar la comprensión de determinado texto a otras personas menos cualificadas.

Esta asimilación entre la actividad de crear literatura y la de traducirla no supone necesariamente que se hubiese producido una disminución de lo que en repetidas ocasiones se ha denominado aquí prestigio social que igualase en la opinión pública medieval ambas ocupaciones. Aunque los dos trabajos se conceptuaban como muy similares, sí que se producían en la mente del hombre medieval diferencias significativas entre el escribir y el traducir que llevaban a una leve desigualdad en la importancia que se le debía asignar, por comparación, a estas dos actividades. Sorprendentemente, se consideraba de mayor valía y creatividad a aquél, a quien hoy se llama traductor, porque su traslación de un texto de un idioma a otro suponía que dominaba más de una lengua, pero, además, que interpretaba a la perfección el mensaje divino contenido en él. Aquél, a quien hoy se califica de autor, sin embargo, adolecía, según se creía, de déficits de preparación cultural, pues, aunque podía captar del mismo modo que el traductor lo escrito por Dios, puede expresarlo sólo en la lengua única que domina.

Lo incongruente de este pensamiento, y la injusticia que encierra para el autor que sí se estime a sí mismo, de algún modo, creativo, ya fue descubierto en la misma Edad Media por algunos escritores orgullosos de sus obras y con conciencia de autor. Konrad von Würzburg, en el siglo XIII, insiste en que los traductores no son poetas ${ }^{11}$, dirigiéndose contra todos aquéllos que se tenían a sí mismos por poetas más doctos, porque, aunque no componían, traducían del latín. Esta queja sin embargo no hace más que confirmar hacia dónde iba la tendencia general. Cabe preguntarse ahora, en vistas de la similitud para el hombre medieval entre crear literatura y traducirla, de qué modo era respetada cualquier actividad de tipo cultural en general.

Recuérdese que se está intentando averiguar si existen razones históricas que justifiquen la idea de que la traducción, a causa de su anonimato y su escaso prestigio en la sociedad, se constituye para ésta como una profesión eminentemente femenina. Durante la Edad Media la invisibilidad del traductor, se ha visto hace un momento, no parece producirse en la medida de lo que cabría esperar, puesto que nombres de traductores medievales pueden citarse muchos, y, además, de ambos sexos indistintamente. En cuanto a la escasa importancia que a la traducción se le pudiera asignar por parte de la sociedad medieval alemana, cualquier tipo de valoración habría de, por necesidad y debido a la concepción del mundo medieval, estar íntimamente relacionada con lo que de la literatura y también de la cultura en general se pensara. Si, en general, la cultura se considerara una actividad menor, y por lo tanto, femenina, también la literatura y la traducción, como actividades de tipo cultural que son, deberán ser desarrolladas preferentemente por mujeres.

\footnotetext{
11 "Es wird betont, daß Übersetzer keine Dichter sind". H. Brinkmann, op. cit., 21
} 
Actividades como saber leer y escribir no eran para el hombre medieval tan comunes y deseables como pudiera parecer hoy en día. Algunos autores medievales, conocidos como creadores de grandes obras literarias, no tienen reparo alguno en reconocer que su cultura es más bien escasa. Wolfram von Eschenbach, por ejemplo, autor de Parzival, la considerada mayor novela épica alemana de su tiempo, presume de no poseer cultura, sin avergonzarse lo más mínimo de ello:

"Wer aber will, daß ich weitererzähle, darf diese Geschichte keineswegs als gelehrtes Buch betrachten. Ich selbst kann nämlich weder lesen noch schreiben.[...] Diese meine Geschichte fügt sich nicht den Grundsätzen gelehrter Schulweisheit. Ehe man sie für ein Buch solcher Art nähme, wollte ich lieber nackt und ohne Badetuch im Bad sitzen" ${ }^{\prime 2}$

Wolfram incluso prefiere que se le tenga por inculto y a su historia como poco erudita, lo cual no dice mucho a favor del concepto que tenía el autor de los sabios y las historias doctas.

Y Wolfram no es el único en jactarse de poseer una educación más bien escasa. Ulrich von Lichtenstein, uno de los trovadores más populares de su época, relata alegremente en Frauendienst, su autobiografía, cómo fue incapaz durante días de averiguar el contenido de un mensaje por escrito que le envió una dama, pues su escriba se había marchado de viaje. Sólo a la vuelta de éste pudo saber que se trataba de un rechazo a su cortejo y desprecio a su persona ${ }^{13}$. El saber leer y escribir no era por lo tanto tan habitual, ni siquiera para los poetas. Bastaba con que éstos tuvieran un escriba que realizara la labor más incómoda para ellos.

En realidad, si se tiene en cuenta que el mundo medieval era, como ya se ha dicho, primordialmente funcional, esto resulta lógico, ya que la utilidad de la cultura para el correcto funcionamiento de ese sistema social era más bien escasa. En un mundo lleno de inseguridades y de desórdenes de todo tipo en el que determinados conocimientos y habilidades podían suponer la diferencia entre la vida y la muerte, no era precisamente leer y escribir lo que más habrá de valorarse. En realidad, sería mucho más lógico si la cultura fuese un campo marginal, al que dedicarse adicionalmente, pero no en exclusiva. Debido a su inutilidad, el presumir de poseer cierta cultura sólo podría resultar prestigioso si iba acompañado de otras muchas cualidades más prácticas y, para ese mundo, más efectivas. Como labor de muy reducido valor social, entonces, sólo aquellos miembros de la sociedad que de por sí poco le aportan en otros campos deberían poder dedicarse a actividades culturales con pleno derecho y, por así decirlo, a tiempo completo: se está hablando ahora de las mujeres.

En efecto, la formación de la mujer solía ser, antes de la aparición de las Universidades, en el campo de la cultura mucho más cuidada que la del varón. Rüdiger Krohn asegura que

\footnotetext{
${ }^{12}$ W. von Eschenbach: Parzival ( Stuttgart: 1981) T 1, 99

13 R. Krohn, "Kulturgeschichtliche Bedingungen" en: H. A. Glaser (ed.), Deutsche Literatur. Eine Sozialgeschichte. Band 1. Aus der Mündlichkeit in die Schriftlichkeit: Höfische und andere Literatur. 750-1320 (Hamburg: 1991) 32
} 
"[...] trotz solcher Benachteiligungen war die Kunst des Lesens und [...] des Schreibens bei den weiblichen Angehörigen des Laienadels verbreiteter als bei den Männern, die über solche Kenntnisse nur in Ausnahmefällen verfügten" ${ }^{\prime 14}$

La influencia de la mujer en el desarrollo de la literatura es muy conocida, Herbert Grundmann por ejemplo, uno de los mayores investigadores de la relación de la mujer medieval con la literatura, está convencido de que

"der Frau als Leserin ebenso die Entstehung einer religiösen deutschen Erbauungsliteratur wie die Aufnahme der deutschen Dichtung in das Schrifttum zu verdanken ist $^{\prime \prime l} 15$

Y, aunque representantes de la actual crítica literaria feminista, como Jürgen Serke, aseguran, ya en el año 1982, que

"Die Literatur, eine Ewigkeit ebenfalls von Männern bestimmt, propagierte in der Bundesrepublik vor einem Jahrzehnt ihr Ende"16

el acceso directo a la literatura de la mujer, es decir, como autora, se produjo en la Edad Media con cierta frecuencia y sin que pudiera considerarse un caso excepcional. Nuevos estudios en esa dirección han demostrado que es posible seguir las huellas de un número sustancioso de autoras alemanas durante la Edad Media, y, por decirlo con las mismas palabras que utilizara Rosamond McKitterick:

"Ich habe bislang keine explizite Äußerung in den Schriften weiblicher Autoren des frühen Mittelalters finden können, die besagt, daß sie einen Boden betraten, der normalerweise oder auch nur gewöhnlich von Männern beschritten wurde"17

Ciertamente, en la literatura no debía tratarse de terreno reservado para uso en exclusiva masculino. Las mujeres, atendiendo a su menor valía social, podían ocuparse de la literatura con toda propiedad, puesto que también esta actividad era de carácter menor en cuanto a consideración social. Y lo que en la Edad Media es apropiado para la literatura creativa, también lo será, puesto que ya se ha visto qué poco se diferencian la una de la otra, para la traducción.

Encontrar con cierta frecuencia nombres de autoras o de traductoras femeninas durante la Edad Media alemana no debe, entonces, sorprender. La literatura y la traducción son en función de su poca importancia para la vida cotidiana medieval, actividades que las mujeres pueden desarrollar con mayor facilidad. El origen histórico de la supuesta adecuación de la mujer para el traducir en base a la escasa trascendencia de la traducción puede deducirse a

\footnotetext{
${ }^{14}$ R. Krohn, ibidem.

${ }^{15}$ H. Grundmann, "Die Frauen und die Literatur im Mittelalter", en: Archiv für Kulturgeschichte, (Leipzig 1936), T 26, 156

${ }^{16}$ J. Serke, Frauen schreiben. Ein neues Kapitel deutschsprachiger Literatur, (Stuttgart 1982) 11

17 R. McKitterick, "Frauen und Schriftlichkeit im frühen Mittelalter" en: H. W. Goetz (ed.), Weibliche Lebensgestaltung im frühen Mittelalter, (Wien 1991) 65-118, aquí 106
} 
partir de la minusvalía de cualquier actividad cultural en general durante la Edad Media como ocupación no marginal, sino principal, y pueden convertir a la traducción, ciertamente ya entonces, en propia de mujeres.

La historia de la traducción tal como la se entiende hoy, y desligada, aunque naturalmente no de forma completa, de la literatura, comienza para Alemania con Lutero ${ }^{18}$. Este divorcio y el desarrollo que por separado han sufrido desde entonces a lo largo de los siglos ambas disciplinas, ha provocado que, en la actualidad, la diferencia de prestigio entre autor y traductor de una obra pueda llegar a ser abismal. A medida que el arte de crear textos ha ido cobrando en importancia a través del tiempo, su tácita aprobación como actividad femenina y su íntima relación con la mujer ha ido retrocediendo. Sólo recientemente la mujer ha vuelto a recobrar una posición dentro la literatura, que, sin llegar a acercarse a la que ostentaba entonces, en la Edad Media podría haber sido suya por naturaleza. Sería interesante observar ahora qué ha sucedido en el campo de la traducción.

La traducción, pese a lo vaticinado en el año 1985 por los que contestaron a la encuesta a la que se ha aludido en un principio, va adquiriendo cada vez más consideración social. La profusión de estudios aparecidos últimamente en torno a la traducción, la existencia de revistas dedicadas en exclusiva a ese tema, los simposios y congresos que recientemente se han celebrado, todo ello indica que la invisibilidad del traductor está a punto de desaparecer para siempre, y que el prestigio social de esta profesión va en aumento. ¿Supone todo esto que, para el futuro, si el nuevo estatus de la traducción se mantiene o incrementa, la mujer perderá ese papel preponderante que la opinión pública aún hace muy pocos años le asignaba, tal como ocurrió en su día con la literatura? Es más, ¿está esto sucediendo ya?

Para ver esta cuestión, se volverá ahora a la estadística y se intentará comprobar cómo se distribuyen por sexos los traductores de las 472 obras que, según el catálogo de la Biblioteca Nacional Alemana, incluían en su referencia bibliográfica el nombre del traductor o traductora. Curiosamente, menos de la mitad de las traducciones que se relacionan han sido efectuadas por mujeres.

En las líneas precedentes se ha estado analizando continuamente la actuación de la mujer en el campo de la traducción en función del prestigio o no prestigio de esta última como disciplina. Como la valoración de las aportaciones de una traducción en concreto depende también en mucho de la importancia que pudiera asignarse a la obra original de la que se ocupa, se han querido estudiar ahora algunas secciones puntuales de entre las obras traducidas, atendiendo a su posible grado de utilidad para el pensamiento social, y ver hasta qué punto son partícipes de la traducción las mujeres.

En primer lugar se han analizado los libros técnicos. Las obras de este tipo, manuales fundamentalmente instructivos, son, esto es innegable, muy útiles para la sociedad, mucho más de lo que pudiera a efectos prácticos aportar un compendio de poesía. Aunque en la escala de valores de entre todos aquellos que han colaborado en la producción y

18 Así lo recogen la mayoría de los estudios sobre historia de la traducción, entre otros: K. Dedecius, Vom Übersetzen, (Frankfurt a .M 1986) 101 
distribución del manual, el traductor sea quizá el que ocupe el lugar más bajo, sin embargo aún presta, aunque indirectamente, un importante servicio. Tal vez por no cumplirse del todo aquí esa 'invisibilidad' de la traducción se explique que sean alarmantemente escasas las traductoras femeninas que puedan encontrarse. Nombres como Eva Verheyen, que tráduce un manual sobre el correcto uso de una bomba a vapor, o Susanne Gorys, que se ocupa de trasladar al alemán una obra compilatoria relacionada con las motocicletas son excepciones que se destacan en un campo que a la mujer le parece vedado. Al perder la traducción de manuales técnicos su etiqueta de actividad poco necesaria, teóricamente, y siguiendo lo que la ya lejana encuesta de Silbermann y Hänseroth postulaba, debería haberse difuminado la preponderancia de la mujer traductora, y, en la práctica, puede verse cómo, en efecto, ha sucedido de ese modo.

Dentro del campo artístico, y, más concretamente, de la literatura, será difícil encontrar un título al que la opinión pública en general califique de más útil que otro, aunque individualmente algún que otro lector tenga a una obra en concreto por tal atendiendo a criterios personales. La utilidad no constituye necesariamente una de las características de lo que hoy se entiende por literatura. De este modo, la estima social de una obra dependerá de diversos otros factores, como el prestigio de su autor, el éxito en ventas, su popularidad para la masa, etc.

Los autores de obras llamadas clásicas, entendiendo aquí clásico en la acepción que le diera Georg Kaiser como fundamentado "en la permanente actualidad con que ejerce su efecto sobre el lector" ${ }^{\prime 1}$, gozarán así de una situación preferente en la escala de valores, puesto que su reputación es en la actualidad y ha sido además históricamente muy respetable. Como de forma usual se trata de autores cuyas obras alcanzan un nivel de complejidad tan notorio como la fama de quien las compuso, la labor de la traducción adquiere una nueva dimensión por su grado de dificultad. Aunque se siga considerando una actividad socialmente poco valiosa, el mérito del traductor se convierte en incuestionable por lo laborioso de su tarea y la especial habilidad que supone. La admiración que a la opinión pública le causa es la misma que se le confiere a todo aquél capaz de realizar alguna curiosidad atrayentemente inútil y dificultosa. Como algo de mérito sí que puede encontrarse para este tipo de traductores, aquí de nuevo el campo se restringe para la mujer, y de tal modo, que fue imposible encontrar una sola traductora femenina para alguna obra compuesta por autores de tanto renombre como James Joyce, Marcel Proust u Honoré de Balzac.

Existe sin embargo un apartado dentro de lo literario en el que las mujeres aún mantienen su hegemonía y en el que dominan por completo, favoreciendo de ese modo que la estadística en cuanto a la distribución en general por sexos de obras traducidas no sea del todo desproporcionada. Se trata de un sector que es de carácter femenino por naturaleza y por definición: la literatura escrita por mujeres. 'Lo que ha escrito una mujer lo puede traducir mucho mejor otra mujer' parecen pensar las autoridades competentes, porque aquí

${ }^{19}$ L. A. Acosta, El lector y la obra. Teoría de la Recepción literaria, (Madrid 1989) 140 
la preponderancia de la mujer es asombrosa. Esto no significa que no sea posible encontrar algún que otro traductor masculino cuya aparición sorprendentemente no tiene por qué estar relacionada de ningún modo con un contenido literario menos univocamente femenino o incluso feminista. Más bien, aquí de nuevo, se atiende al prestigio de la autora. Es posible, por ejemplo, encontrar algunos traductores masculinos que se ocupen de la obra de Virginia Woolf, que suele ser de contenido mucho más cercano a lo que se comprende como mundo exclusivamente femenino, y a la que se conoce por su marcado carácter feminista. La proporción en número de los traductores varones para Virginia Woolf, cinco, frente a doce traductoras de sexo femenino, es bastante desigual, pero aún así todavía respetable.

Algo muy similar ocurre con la prestigiosa autora norteamericana Doris Lessing. A pesar de que sus obras tratan sobre todo de plasmar una visión más bien femenina de la realidad y aunque la mujer mantiene una posición fundamental dentro de sus escritos, la cifra de traductores varones que se han ocupado de trasladar sus obras al alemán es idéntica a la que se ha podido constatar para Virginia Woolf, es decir, cinco, frente a en este caso, once traductoras mujeres. En ambas ocasiones se llega a resultados muy similares.

A medida que la popularidad de la autora y el concepto que de la calidad de su obra tiene el público en general desciende, los traductores masculinos disminuyen a su vez. Autoras que, por diferentes razones, se encuentran en algo así como un escalafón intermedio y cuya valía no está del todo probada, o, al menos no es universal o unánimemente aceptada, son capaces todavía de presumir de uno o dos traductores masculinos aislados, pero nunca más. Ejemplos interesantes que pueden situarse dentro de este grupo lo constituyen la autora de color Alice Walker y la llamada 'dama del crimen' Agatha Christie.

En el caso de Alice Walker, su posición intermedia en la escala de valores, pese a contar en su haber con un Premio Pulitzer en el apartado de Ficción por su obra El color púrpura, más conocida en su adaptación cinematográfica por el director norteamericano de éxito Steven Spielberg, se debe a su juventud, además de no ser una autora muy prolífica en obras. La calidad de sus escritos aún está por determinar y no ha sido hasta ahora cuando internacionalmente ha empezado a ser conocida. En cuanto a Agatha Christie, el escaso prestigio del que gozan por lo general las novelas de tipo detectivesco de las que es autora, y la facilidad con la que parece producirlas, la convierten en una escritora controvertida. Si hay o no calidad literaria en sus obras no está muy claro, pues no acaba de producirse el acuerdo. Así, para sus nada menos que ciento veintidós obras traducidas al alemán en el período estudiado, sólo tres son los traductores masculinos mencionados.

Aunque aquí el número sí que parece del todo desnivelado, aún más evidente resulta la división en sexos de las obras traducidas en aquéllas que se consideran escasamente literarias. Son ese tipo de novelas llamadas góticas, compuestas como una mezcla de lo romántico y lo misterioso, que gozan de un enorme favor entre el público, se supone que sobre todo femenino, lo cual se traduce en un éxito en ventas que sería la envidia de cualquier autor clásico. El prestigio de la autora, que en este caso no puede identificarse con su fama, es prácticamente nulo. En ocasiones estos escritos incluso se llegan a considerar como subliteratura, y la elaboración de su trama es tenida por pobre y repetitiva. Se trata de 
literatura de simple entretenimiento, cuya calidad artística no ha de ser necesariamente muy elevada. Un ejemplo interesante dentro de este apartado es la autora inglesa Victoria Holt. Sus novelas han sido traducidas a numerosos idiomas y sólo en el período estudiado se han publicado en la República Federal de Alemania nada menos que sesenta obras suyas traducidas al alemán. Para éstas, ninguna de las cuales parece destacar significativamente sobre otra, no se ha contado con un solo traductor masculino.

Es decir, que se ve, cómo efectivamente, y tal como la encuesta popular realizada en Colonia en el año 1985 parecía sugerir, a menos calidad de la obra según la opinión pública, a menos importancia asignada a la traducción en la consideración social, mayor probabilidad habrá de que de la traducción sea realizada por una traductora femenina, independientemente de si el tema que se trate interese más o menos o se ocupe en mayor o menor medida de las mujeres.

En las líneas que anteceden ha podido comprobarse cómo históricamente en toda actividad cultural podía participar libremente en especial la mujer, puesto que tanto la cultura como la mujer ocupaban un lugar menor dentro del desarrollo social. Hoy en día la cultura es deseable, aunque no necesariamente útil, para un sector social mucho más amplio, y aunque en teoría la mujer ocupa ya una posición similar o igual a la del varón, en la práctica su labor parece seguir siendo la de desarrollar tareas consideradas minoritarias, lo cual en concreto en la traducción significa, según se ha visto, trasladar al alemán únicamente obras de menor valía. La mujer parece haber vuelto en ese caso en cierto modo a ocupar posiciones que le asignaba la sociedad medieval, o tal vez sea que la sociedad medieval y la actual no se diferencian tanto como pretenden. Como la presencia de la mujer desaparece en aquellos sectores de la traducción que sí pudieran aportarle algún prestigio, con la mayor estima de la actividad del traducir, que convierte a ésta en una disciplina útil y a veces hasta en artística por sí misma, la participación de la mujer tiende a desaparecer. Si la traducción evoluciona, como parece, de modo similar a como lo hiciera en su día la literatura, dejando de ser una ocupación marginal, para convertirse en prestigiosa, no sería tan extraño que la mujer quedase relegada para siempre a un segundo término, y se ocupara, más aún de lo que viene haciendo ahora, de temas que socialmente se consideran marginales y menores. En este sentido, será necesario reflexionar, sobre la base de lo que aquí y ahora se ha expuesto, si la traducción es y ha sido para la mujer a lo largo de la historia y también en este mismo momento una vocación libre y natural o no se habrá visto de algún modo forzada. 